\title{
Using Topic Maps in an E-learning Context
}

Marie-Hélène Abel, Dominique Lenne, Claude Moulin, and Ahcene Benayache

UMR CNRS 6599, BP 20529

60205 Compiègne cedex, France

$+33(0) 344234950$

\{Marie-Helene.Abel, Dominique.Lenne, Claude.Moulin, Ahcene.Benayache\}@utc.fr

\begin{abstract}
E-learning leads to evolutions in the way of designing a course. Diffused through the web, the course content cannot be the pure transcription of a face to face course content. A course unit can be seen as an organization in which different actors are involved. These actors produce documents, information and knowledge that they often share. Within the MEMORAe ${ }^{1}$ project [1] we design an ontology-based document-driven memory which we think as being particularly adapted to an e-learning situation. We precise the choice of the Topic Maps formalism that we use for knowledge representation.
\end{abstract}

\section{A Learning Organizational Memory}

A course unit is based on knowledge and competencies it should provide, on actors (learners, instructors, trainers, course designers, administrators, etc.) and on resources of different types (definitions, exercises with or without solution, case studies, etc.), and different forms (reports, books, web sites, etc.). In this sense, a course is an organization. A common approach to tackle the knowledge management problem in an organization consists in designing an organizational memory [2]. In order to share information in an organization, actors have to use a common terminology, especially when they are geographically distant. A given word or expression must have the same meaning for everyone. It is one of the reasons why organizational memories are often based on ontologies.

The learning organizational memory we propose aims at facilitating knowledge organization and management for a given course or training, and at clarifying competencies it allows to acquire.

\section{The Choice of the Topic Maps Formalism}

We have to consider two levels for modeling the memory and building ontologies: the first one is generic; the other one is specific and is relative to a particular training program. These two levels are both used for document and resource indexing and for

\footnotetext{
1 The MEMORAE project is supported by STEF, a research pole of the Picardie region. 
helping to navigate between the knowledge elements of the memory that serve to index the documents.

The modeling of a training memory contains three elements: two ontological parts and the way to index documents on them. The modeling must at least allow three operations: (1) The reunion of two ontologies: the generic one and the application one; (2) The substitution of an application ontology by another one coming from another domain; (3) The attachment of document indexing on the reunion of two ontologies.

The choice of the formalism(s) for representing the memory is very decisive. It must go beyond the hybrid aspect of the modeling (ontology and indexing), favor the interoperability between various tools that have to deal with the memory (edition, updater, consultation, navigation, etc.).

The Topic Maps formalism (TM) [3] is useful to define and manipulate the information attached to resources. That provides a logical organization to a large quantity of resources keeping them accessible and facilitating the navigation between them.

Overall, we chose the TM formalism because it keeps a semantic level close enough to the model of our memory. With an ontology oriented point of view, this formalism allows to envisage the important following characteristics:

- It is possible to consider some topics as generic concepts and other as concept instances;

- It is possible to consider associations, scopes and occurrences as roles between concept topics;

- Associations have no limitation in their member number;

- The occurrence relation allows to directly attach resources to concepts (the same resource can appear in several occurrence relations and be accessible from more than one concept);

- Relations (associations, occurrence) and concept labels can be defined inside scopes. This allows to simply implementing annotations (or points de view in the memory).

To definitely adopt this formalism, we verified it was possible to simply add ontological missing features and mainly the relation superclass-subclass for building hierarchies of concepts.

\section{References}

1. Abel M.-H., Lenne D., Cissé O., "E-Learning and Organizational Memory", Proceedings of IC-AI'02, Las Vegas, June 2002.

2. Rabarijaona A., Dieng R., Corby O., Ouaddari R., Building a XML-based Corporate Memory, IEEE Intelligent Systems, Special Issue on Knowledge Management and Internet, p. 56-64, May-June 2000.

3. IEC, "International Organization for Standardization (ISO)", International Electronical Commission (IEC), Topic Map, International Standard ISO/IEC 13250, 19 April 1999. 